\title{
Stable operation of a synchronously pumped colliding-pulse mode-locked ring dye laser
}

\author{
M. C. Nuss, R. Leonhardt, and W. Zinth \\ Physik Department der Technischen Universität München, München, Federal Republic of Germany
}

Received August 10, 1984; accepted October 22, 1984

\begin{abstract}
Pulses of 100-fsec duration are obtained by synchronous pumping of a colliding-pulse ring dye laser with a modelocked $\mathrm{Ar}^{+}$-ion laser. Stable operation of the synchronously pumped colliding-pulse mode-locked laser over hours was obtained by a suitable choice of the distance between the gain and the absorber in combination with an appropriate pump-pulse sequence.
\end{abstract}

Passive mode locking of a ring dye laser by the interaction of two counterpropagating pulses in a thin saturable absorber (colliding-pulse mode locking) yields femtosecond laser pulses. ${ }^{1}$ In these lasers the gain medium (Rhodamine 6G) is pumped by a cw $\mathrm{Ar}^{+}$-ion laser. The saturable absorber (DODCI, 3,3-diethyloxadicarbocyanine iodide) synchronizes two counterpropagating pulses meeting in the absorber jet stream. The colliding pulses form a transient grating, which synchronizes and stabilizes the pulses. ${ }^{2}$ In order to ensure equal amplification for both counterpropagating pulses, the distance between the absorber and the gain medium should be close to one quarter of the ring perimeter $L$.

The cw pumped colliding-pulse mode-locked (CPM) laser has two shortcomings: (1) There is no second ultrashort light source synchronizable to the CPM laser at a second frequency. Even a synchronized picosecond source would be helpful for certain excite-and-probe experiments. The standard way to overcome this problem is to use amplified pulses and continuum generation, requiring elaborate equipment that leads to a low-repetition-rate system. (2) The second harmonic of a cw mode-locked Nd:YAG laser as a pulsed source cannot be used to pump a standard CPM ring laser.

In order to overcome these difficulties several attempts have been made to pump femtosecond dye lasers synchronously: (1) Mixing the absorber and the gain dyes in a single jet yields subpicosecond pulses: 70 -fsec pulses have been reported for this system. ${ }^{3}$ Other authors could obtain pulses of 200 -fsec duration with insufficient coherency properties for the same laser setup. $^{4}$ (2) Recently, a standard-type CPM laser was pumped at twice its round-trip rate $\omega_{R}=1 / T_{R}$ by the second-harmonic pulses of a cw mode-locked Nd:YAG laser. ${ }^{5}$ Unfortunately, this system requires an absorber-gain distance of exactly one quarter perimeter. This leads-in the case of synchronous pumping at $2 \omega_{R}$-to a bistable operation of this laser, as two pairs of counterpropagating pulses can build up in the laser. ${ }^{5}$

In this Letter we discuss a CPM laser synchronously pumped by a suitably arranged sequence of pump pulses. The distance $D$ between the absorber and the amplifier of the CPM laser is set to approximately one fifth the cavity perimeter $L, D=1 / 5 L$. Since the dye-laser pulses generated by two subsequent pump pulses should collide in the absorber jet, the gain medium must be pumped at times $t=0,3 / 5 T_{R}, T_{R}, 8 / 5$ $T_{R}, 2 T_{R}, \ldots$ We designed the pumping system in the following way (see Fig. 1, dashed lines): A cw modelocked $\mathrm{Ar}^{+}$-ion laser with the same cavity round-trip time $T_{R}$ as the ring laser supplies pulses $\mathrm{P} 1$ that enter the gain jet at $t=0, T_{R}, 2 T_{R}, \ldots$ The second train of pump pulses (P2), transmitted through the beam splitter BS and delayed in the optical delay line, arrives at the gain medium at $t=3 / 5 T_{R}, 8 / 5 T_{R}, \ldots$ All pulses are focused into the gain jet by mirrors of 150-mm focal length.

The delay of $3 / 5$ round-trip time in the gain medium between the two pump pulses guarantees that only the dye-laser pulses resulting from pump pulses with $3 / 5$ $T_{R}$ delay with respect to one another collide in the absorber jet stream and form a transient grating. The absorption recovery time of DODCI ( $1.1 \mathrm{nsec})$ (Ref. 6) ensures that the other two pulses, which may also be generated in the gain medium, do not meet in the absorber and thus experience a higher loss and are suppressed.

The solid lines in Fig. 1 represent the dye-laser path in the CPM laser cavity. A standard jet nozzle of $350-\mu \mathrm{m}$ thickness is used for the $6 \times 10^{-4} \mathrm{M}$ solution of Rhodamine $6 \mathrm{G}$, whereas the $5 \times 10^{-3} \mathrm{M}$ DODCI absorber has $20-\mu \mathrm{m}$ thickness. The radius of curvature is $100 \mathrm{~mm}$ for mirrors M1 and M2 and $50 \mathrm{~mm}$ for M3 and M4. All other mirrors are flat. The transmission of the output coupler is $2.5 \%$. The output coupler is mounted on a translation stage, so that the cavity length can be adjusted to match the pump-laser repetition rate. Care has to be taken to keep the cavity length constant.

The pulses of our CPM laser were analyzed by four techniques: (1) With a fast photodiode and an oscilloscope, we monitored the stability of the pulse train. (2) The autocorrelation trace of the pulses was mea- 
sured by background-free second-harmonic generation in a KDP crystal of $150-\mu \mathrm{m}$ thickness. ${ }^{7}$ The overall glass path in the autocorrelator is less than $5 \mathrm{~mm}$. (3) With a similar setup we recorded the cross-correlation trace of the two counterpropagating light pulses from the CPM laser. (4) The spectrum was measured by a grating spectrometer (resolution $0.4 \mathrm{~nm}$ ).

Stable lasing of the CPM laser can be obtained at a pump level of the $\mathrm{Ar}^{+}$laser of $500 \mathrm{~mW}$. Good mode locking and stable operation of the $\mathrm{Ar}^{+}$-ion laser is required. The cavity length of the dye laser must be adjusted with high precision. Without a perfect match of the cavity lengths of the $\mathrm{Ar}^{+}$and CPM lasers, strong spiking, relaxation oscillation, and unidirectional laser action is observed for the CPM laser. When the cavity length is correctly set, stable laser action is found for the two counterpropagating pulses in the laser. In order to have good operation at the shortest pulse durations, the optimum cavity length has to be maintained within better than $0.2 \mu \mathrm{m}$. Under these conditions the pulse trains are stable with a small ripple of $5 \%$ rms at an emitted power of $28 \mathrm{~mW}$. The temporal properties of the pulses are shown in Fig. 2. The width of the autocorrelation trace (Fig. 2@) is 170 fsec, indicating a pulse duration of $110 \mathrm{fsec}$ assuming sech $^{2}$ pulses. Precise alignment of the laser permitted generation of pulses shorter than $100 \mathrm{fsec}$. The autocorrelation trace is smooth and shows no indication of a coherency spike. We have also measured the autocorrelation trace over more than 3 orders of magnitude. The trace decays almost exponentially, consistent with a sech ${ }^{2}$ shape.

Even at a factor of 1000 below the peak, there is no

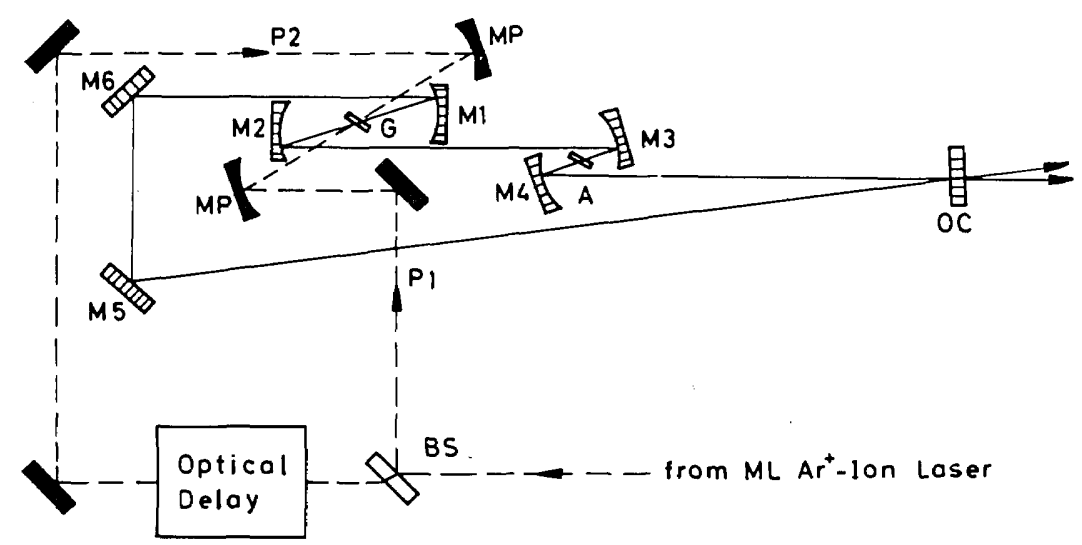

Fig. 1. Schematic diagram of the synchronously pumped CPM ring dye laser. The ring laser (hatched mirrors) consists of focusing mirrors M1-M4, flat mirrors M5 and M6, a 2.5\% transmission output coupler OC, a gain jet G, and an absorber dye jet A. The pump geometry (filled mirrors) is indicated by the broken lines. The pump beam is divided into two parts of equal power by the beam splitter BS. The reflected beam is focused (mirror MP) directly into the gain jet, whereas the transmitted beam first passes the optical delay line and is then focused into the gain dye jet.

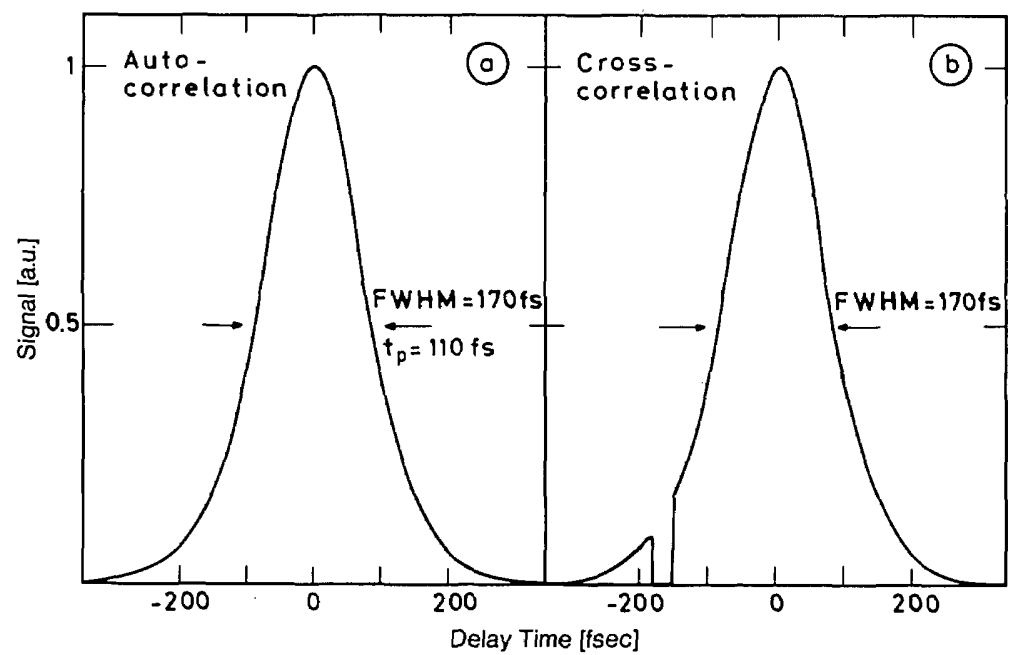

Fig. 2. (a) Autocorrelation trace of pulses from the synchronously pumped CPM dye laser. FWHH, 170 fsec. A pulse duration of $110 \mathrm{fsec}$ is calculated assuming sech ${ }^{2}$-shaped pulses. (b) Cross-correlation trace of the counterpropagating pulses from the synchronously pumped CPM laser. The curve has the same width of $170 \mathrm{fsec}$ as the autocorrelation trace, demonstrating the perfect synchronization of the two counterpropagating pulses. 


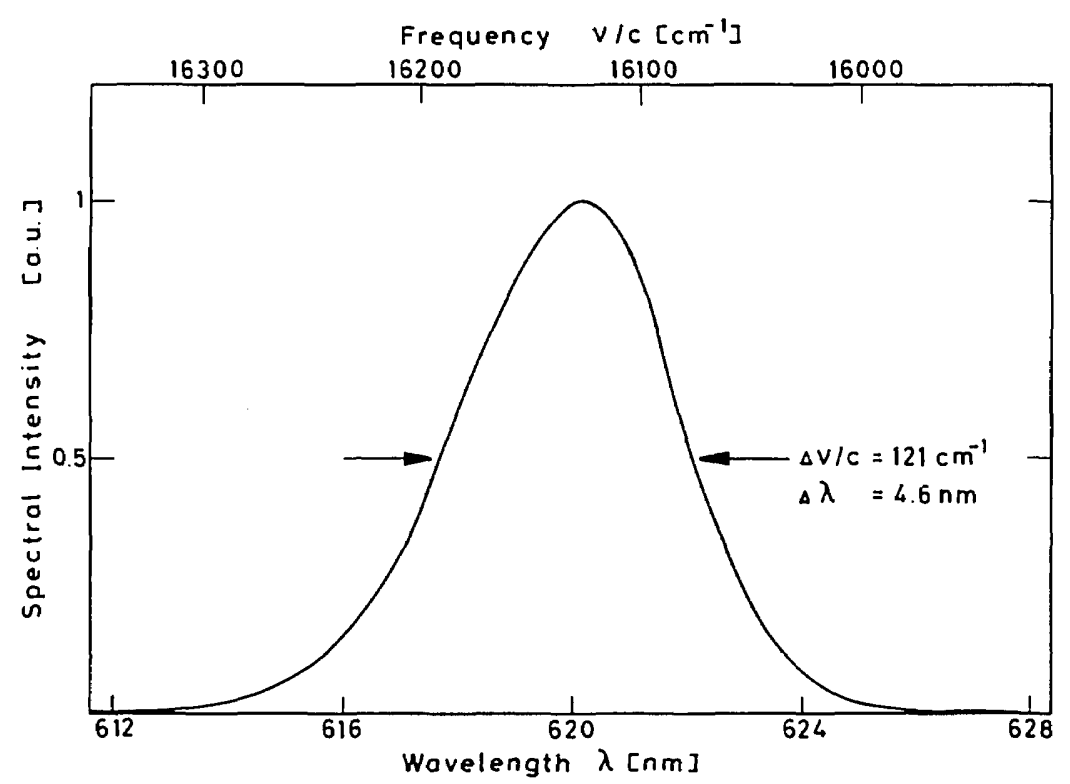

Fig. 3. Spectrum of the dye-laser pulses from the synchronously pumped CPM laser. The bandwidth product $t_{p} \Delta \nu=0.4$ is close to the value 0.315 for $\operatorname{sech}^{2}$-shaped pulses.

indication of background emission. Interesting results on the CPM mechanism are obtained from the crosscorrelation trace (see Fig. 2(b)) between the two counterpropagating pulses: The cross-correlation trace has the same width of $170 \mathrm{fsec}$ and a very similar shape to the autocorrelation trace. This demonstrates that the two pulses are indeed synchronized by the absorber and that they have negligible jitter.

Figure 3 depicts the spectrum of the femtosecond pulses. The spectral width of $4.6 \mathrm{~nm}$ leads to a bandwidth product of 0.4 , which is close to the transformlimited value of 0.315 for $\mathrm{sech}^{2}$-shaped pulses. The asymmetry of the spectrum shows that the dye-laser pulses have a slight chirp. A compensation of the chirp might lead to even shorter pulses. ${ }^{8}$

Interesting information is obtained when the same CPM laser configuration is pumped by a cw, nonmode-locked $\mathrm{Ar}^{+}$-ion laser at 5 -W pump power. We removed the beam splitter in the pump scheme and shortened the cavity length by shifting the output mirror, so that the absorber-gain distance is $\sim 1 / 4 L$. This cw-pumped CPM laser gives results similar to the synchronously pumped laser: The autocorrelation trace exhibits the same duration of the laser pulses as in the system discussed above. In addition, a similar output power of $35 \mathrm{~mW}$ is obtained. This indicates that the pulse duration in the synchronously pumped CPM laser is probably limited by the bandwidth and dispersion of the optical elements in the resonator and not by the synchronous pumping scheme. Thus further shortening of the synchronously pumped CPM laser pulses seems possible by introducing appropriate dispersion elements into the resonator.

We have demonstrated a modified colliding-pulse femtosecond dye laser ( $t_{p} \simeq 100 \mathrm{fsec}$ ), which can be pumped synchronously by a mode-locked $\mathrm{Ar}^{+}$-ion or a frequency-doubled Nd:YAG laser. The stable operation makes the synchronously pumped CPM laser well suited for experimental applications, e.g., optical gate experiments, in which the synchronization with a tunable picosecond laser would permit new experiments with femtosecond time resolution.

The authors acknowledge valuable contributions of W. Kaiser.

\section{References}

1. R. L. Fork, B. I. Green, and C. V. Shank, Appl. Phys. Lett. 38, 671 (1981); W. Dietel, J. J. Fontaine, and J. C. Diels, Opt. Lett. 8, 4 (1983).

2. E. M. Garmire and A. Yariv, IEEE J. Quantum Electron. QE-3, 222 (1967); M. S. Stix and E. P. Ippen, IEEE J. Quantum Electron. QE-19, 520 (1983); D. Kühlke, W. Rudolph, and B. Wilhelmi, IEEE J. Quantum Electron. 19, 526 (1983).

3. G. A. Mourou and T. Sizer II, Opt. Commun. 41, 47 (1982).

4. J. Kluge, "Synchronously pumped dye lasers for ultrashort light pulse generation," thesis (Gesamthochschule Essen, Essen, Federal Republic of Germany, 1984).

5. A. M. Johnson and W. M. Simpson, Opt. Lett. 8, 554 (1983).

6. D. Magde and M. W. Windsor, Chem. Phys. Lett. 27, 31 (1974); W. Sibett, J. R. Taylor, and D. Welford, IEEE J. Quantum Electron. QE-17, 500 (1981).

7. M. Maier, W. Kaiser, and J. A. Giordmaine, Phys. Rev. Lett. 17, 1275 (1966); H. P. Weber, J. Appl. Phys. 38, 2231 (1967).

8. C. C. Shank, R. L. Fork, and R. T. Yen, in Picosecond Phenomena III, Vol. 23 of Springer Series in Chemical Physics, K. B. Eisenthal, R. M. Hochstrasser, W. Kaiser, and A. Laubereau, eds. (Springer-Verlag, Heidelberg, 1982), pp. 2-5; J. J. Fontaine, W. Dietel, and J. C. Diels, IEEE J. Quantum Electron. QE-19, 1467 (1983). 